\title{
Konsumentenschutz durch das AGB-Gesetz?
}

Die offizielle Verbraucherpolitik in der Bundesrepublik Deutschland ist gekennzeichnet durch die Elemente Markt- und Informationspolitik: Verbraucherschutz soll sich in marktwirtschaftlichen Systemen über wirksamen Wettbewerb und die informierte Wahrnehmung von Marktpositionen (Nachfrage) realisieren. Verbraucherpolitik ist demnach im Kern Wettbewerbspolitik, an der Peripherie auf diese bezogene Informations- und Beratungspolitik, die rechtlich abgesichert werden. Wettbewerbsrecht dient einem mittelbaren Verbraucherschutz durch Erhaltung von Anbieterkonkurrenz, rechtlich durchgesetzte Information der rationalen Wahrnehmung von Marktchancen durch die Konsumenten. Beides soll die Voraussetzungen des Funktionierens marktwirtschaftlicher Systemstrukturen schaffen.

Über diese beiden Momente hinaus stellt sich das Problem Konsumentenschutz dann nur unter dem Gesichtspunkt des Mißbrauchs von Marktmachtpositionen, wenn also das Primärziel wettbewerbsrechtlicher Kontrolle, nämlich wirksamen Wettbewerb herzustellen, nicht realisiert wurde. Prinzipiell mit einem solchen Ansatz rechtlicher Kontrolle vereinbar ist eine privatrechtlich orientierte Rechtskonzeption, die nicht im weiten Sinne Äquivalenzprobleme entscheidet, sondern sich auf die wettbewerblich und wettbewerbsrechtlich kontrollierte private Autonomie einläßt und an der Vertragskategorie als einer auf subjektiven Willen bezogenen festhält.

Insbesondere die durch die Rechtsprechung entwickelten Kontrollen von allgemeinen Geschäftsbedingungen entsprechen dieser Konzeption. Voraussetzung der dort vollzogenen Inhaltskontrolle ist der Mißbrauch von Marktpositionen, Kriterien der Entscheidung entstammen dem dispositiven Recht, das damit zu zwingendem Recht umgestaltet wird.

\section{Zum materiellrechtlichen Programm des AGB-Gesetzes}

Das Gesetz zur Regelung des Rechts der allgemeinen Geschäftsbedingungen (AGBGesetz) vom 9. I 2. 1976', das am r. 4. 1977 in Kraft tritt, nimmt die privatrechtliche Konzeption auf. Im Kern übernimmt es die von der Rechtsprechung entwickelten Entscheidungsregeln, hebt die inter-partes-Wirkung, die die Entscheidungen jedenfalls rechtlich bisher nur entfalten konnten, zugunsten einer Verallgemeinerungswirkung auf. Das Gesetz unterscheidet - wie schon die Rechtsprechung - indirekte Inbaltskontrolle, als Kontrolle der Einbeziehung in den Vertrag $(\mathbb{S} 2)^{2}$ und Kontrolle durch Auslegungsregeln ( $\mathbb{3} 3$ überraschende Klauseln, $\mathbb{S}$ Unklarheitenregeln) und direkte in der Form der Generalklausel ( $\$ 9$ ), die sich auf den Gerechtigkeitsgehalt von Normen und die Vertragszwecke bezieht, sowie durch Klauselverbote mit und ohne Wertungsmöglichkeit ( $\mathbb{S}$ IO, I I). Diese Kontrollnormen werden durch ein allgemeines Umgehungsverbot $(\$ 7)$ abgesichert.

$\$ 2$ AGB-Gesetz weist deutlich auf die privatrechtliche Orientierung hin: AGB können Vertragsinhalt nur werden,

»wenn der Verwender bei Vertragsabschluß

1) die andere Vertragspartei ausdrücklich oder, wenn ein ausdrücklicher Hinweis wegen der Art des Vertragsabschlusses nur unter unverhältnismäßigen Schwierigkeiten möglich ist, durch deutlich sichtbaren Aushang am Ort des Vertragssschlusses auf sie hinweist und

I BGBl. I Seite 3317 von 1976

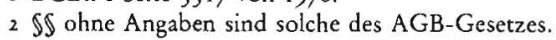


2) der anderen Vertragspartei die Möglichkeit verschafft, in zumutbarer Weise von ihrem Inhalt Kenntnis zu nehmen,

und wenn die andere Vertragspartei mit ihrer Geltung einverstanden ist."

Dieser letzte Halbsatz des $\$ 2 \mathrm{I}$ ist gegenüber dem Entwurf der Bundesregierung vom 6. 8. $1975^{3}$ durch die Betonung des Willenserklärungscharakters des Einverständnisses verschärft worden. Im $\mathbb{2}$ I des Regierungsentwurfes hieß es an dieser Stelle:

"... wenn das Verhalten der anderen Vertragspartei den Umständen nach als Einverständnis mit ihrer Geltung angesehen werden kann «, eine Formulierung, die weniger auf Willensmomente, denn auf objektivierte Zurechnung und damit auch auf die Erwartungen des Verwenders von AGB aus ist. Die jetzige Betonung von Willensmomenten eröffnet auch gegenüber der bisherigen Rechtsprechung des BGH eine erweiterte Chance für verdeckte Inhaltskontrolle. Die gleiche Orientierung zeigt $\S_{4}$ (Vorrang der Individualabrede), der mündliche Nebenabreden unter bestimmten Umständen zuläßt. Dieser Tendenz, am Willensprinzip festzuhalten, steht eine umfangreiche Skala von einzelnen Klauselverboten gegenüber, die eine weitgehende Festlegung von traditionell privater Autonomie zugerechneter Vertragsinhaltsgestaltung in Form der AGB vorsehen. Kontrolle stellt sich dann dar als Ausschluß des Anknüpfungspunktes "Willen « auf der Verwender-/Anbieterseite und Betonung dieses Anknüpfungspunktes auf der Konsumenten-/Nachfragerseite, beides aber orientiert am Vertragsmodell, in dem gleich mächtige Willenssubjekte Kompromisse aushandeln. Rechtliche Kontrolle stellt sich insofern ausschließlich als Wiederherstellung von vertragsrechtlicher gleicher Berechtigung auf der Ebene der Willensautonomie dar. Auf der anderen Seite werden aber durch die $\$ \$ 9_{9-1} 1$ Vertragsäquivalente festgesetzt. Die alte Fiktion vom Vertrag als Richtigkeitsgewähr (= private Autonomie) wird aufgegeben zugunsten einer gesetzlich bzw. richterlich verwalteten Vertragsäquivalenz, deutlich insbesondere in den Klauselverboten des $\mathbb{I}$ I . Die Grenze solcher Materialisierung des Privatrechts wird aber sogleich in $\$ 8$ (Schranken der Inhaltskontrolle) bezeichnet: Nur solche AGB sind der direkten Kontrolle unterworfen,

»durch die von Rechtsvorschriften abweichende oder diese ergänzende Regelungenvereinbart werden."

Die Begründung des auch insofern vom Bericht des Rechtsausschusses übernommenen $\$ 6$ des Regierungsentwurfes lautet:

"Die Leistungsbeschreibung einschließlich etwaiger in AGB enthaltener Festlegungen des Entgelts unterliegen der Inhaltskontrolle danach ebensowenig wie AGB, die lediglich den Inhalt gesetzlicher Regelungen wiedergeben. Denn aufgrund der Inhaltskontrolle nach den

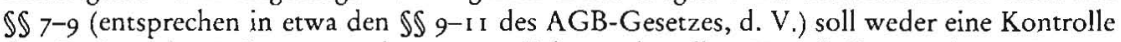
der Preise oder Leistungsangebote ermöglicht noch sollen Vorschriften anderer Gesetze modifiziert werden. $\varkappa^{4}$

Eine Qualitäts- oder Preiskontrolle soll/darf nicht Gegenstand privatrechtlicher, auf Marktmechanismen bezogener Regelung sein. Preiskontrolle ist als kartellrechtliche Mißbrauchskontrolle vom Bundeskartellamt ${ }^{5}$ und vom Kammergericht ${ }^{6}$ zwar in jüngeren Entscheidungen zum Arzneimittelmarkt in Anspruch genommen, vom Bundesgerichtshof ${ }^{7}$ abstrakt akzeptiert, konkret (Abgrenzung des relevanten Mark-

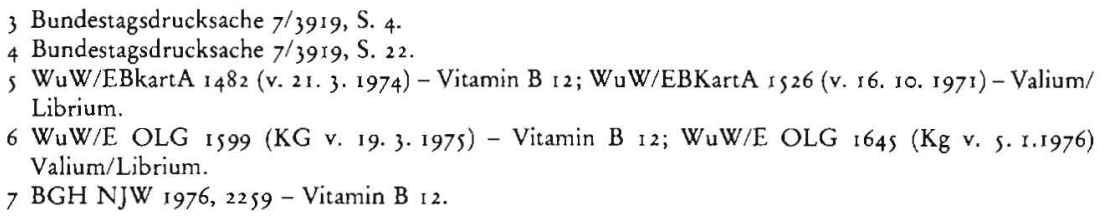


tes) in der Vitamin B I 2-Entscheidung abgelehnt worden; ihre verfassungsrechtliche Legitimation ist bestritten. ${ }^{8}$ Qualitätskontrolle findet bisher meist nur als Informationspflicht über Qualität oder als Selbstkontrolle statt.

An dieser Stelle wird die Ambivalenz der durch das Gesetz gewählten Lösung sichtbar: Einerseits in das Vertragsgefüge durch zwingende Kontrollnormen eingreifen, sich damit aber andererseits notwendig von der privatrechtlichen Konzeption entfernen zu müssen und der Rechtsprechung umfangreiche Kompetenzen zugunsten der Herstellung von justiziell angeleiteten privatautonomen Handlungschancen auf der Konsumentenseite einzuräumen, die aber ihrerseits wiederum begrenzt sind, obwohl gerade Preis und Qualität und damit die Gebrauchswertqualität der Produkte Verbraucherinteressen treffen. Sie betreffen aber auch andere wirtschaftspolitische Zielsetzungen, etwa die des ständigen Wirtschaftswachstums oder die der Arbeitsplatzerhaltung.

Kennzeichnend für diese Materialisierungstendenzen ist also ein punktueller Interventionismus, der immer neue Aspekte und Materien des Marktgeschehens reguliert und in Rechtsprechung überführt und somit zivilrechtliche Autonomie, insbesondere Handlungsalternativen auf der Anbieterseite durch zwingende Anforderungen begrenzt, ohne daß allerdings eine einheitliche wirtschaftsrechtspolitische Orientierung erkennbar würde. Die Erweiterung des Entscheidungsrahmens der Justiz, die Schaffung "zwingenden Zivilrechts« durch den Gesetzgeber benennen aber bisher nur das Dilemma, daß das Zivilrecht seinen Status als Formalrecht noch nicht überwunden, seine Auflösung in Wirtschaftsrecht, das auf sozialstaatlich angeleitete politische Planung des ökonomischen Systems abzielt, noch nicht erreichen konnte.

\section{Zum Verfabrensprogramm}

Der verfahrensrechtliche Teil zeigt erneut die Ambivalenz: Das, was an Materialisierung auf dem materiellrechtlichen Gebiet zugestanden ist, wird, da nicht justiziell vorprogrammiert, im verfahrensrechtlichen Bereich nicht mehr eingelöst, da das Risiko der Rechtsverfolgung weit- und weiterhin privatisiert bleibt. Der Ansatz einer Entprivatisierung/Veröffentlichung der Rechtsverfolgung und Anwendungskontrolle durch quasi-öffentliche Uberwachungsinstanzen wird nicht konsequent verfolgt.

Im Entwurf der Bundesregierung war eine verfahrensrechtliche Regelung noch nicht vorgesehen. Die jetzige Fassung folgt der Intervention des Bundesrates, der sich von zwei Gesichtspunkten leiten ließ: Dem der marktwirtschaftlichen Verfassung der Wirtschaftsordnung und dem der Systematik des geltenden Rechts. ${ }^{9}$ Der erste spricht gegen staatlichen Dirigismus = Uberwachung der Verwendung von AGB durch Verwaltungsbehörden, der zweite für eine Unterlassungsklage und gegen ein Prüfungsverfahren nach Art einer abstrakten Normenkontrolle.

Das Gesetz gewährt gegen unwirksame AGB ( $\mathbb{S}$ 9-1 I) einen Unterlassungs- und Widerrufsanspruch, der von Verbraucher- und anderen Verbänden $(\$ 13)$ geltend gemacht werden kann. Die Landesregierungen werden ermächtigt, besondere Kammern bei einem Landgericht für die Bezirke mehrerer Landgerichte einzurichten, bei dem sämtliche Verfahren konzentriert werden ( $\$$ I 4). Die Urteilsformel ( $\$$ I7) kann $(\mathbb{I} 8)$ im Bundesanzeiger veröffentlicht werden und wird von Amts wegen

\footnotetext{
8 Hans Peter Ipsen, Kartellrechtliche Preiskontrolle als Verfassungsfrage, Baden-Baden 1976 mit weiteren Angaben.

9 Bundestagsdrucksache $7 / 3919$, S. 54 f.
} 
dem Bundeskartellamt zur Eintragung in ein Register mitgeteilt $(\$ 20)$, über die jedermann auf Antrag Auskunft zu erteilen ist. Die Wirkung des Unterlassungsurteils ist auf das Unterlassungsverfahren begrenzt, sofern nicht ein weiterer Betroffener sich in einem anderen Verfahren auf die dort ausgesprochene Unwirksamkeit beruft.

Während die materiellrechtlichen Normen allgemeine Festlegungen enthalten, wirkt das Urteil nach $\$_{13}$ lediglich inter-partes, seine Verallgemeinerungswirkung ist abhängig vom Antrag der betroffenen Partei. Die Verallgemeinerungswirkung tritt also nur auf Antrag und nur bezogen auf denselben Verwender/Aufsteller der AGB ein, nicht etwa bezogen auf die unwirksamen Bedingungen.

„Die Breitenwirkung beschränkt sich auf Individualprozesse mit einem verurteilten Verwender. In allen anderen Fällen bleibt es bei dem allgemeinen Grundsatz, daß das Gericht die Wirksamkeit von Bestimmungen in $\mathrm{AGB}$ zu prüfen hat. "10

Damit ergibt sich eine doppelte Diskrepanz zwischen materiellrechtlichem und verfahrensrechtlichem Programm: Während dort allgemeine Regelungen zur Effektivierung von Verbraucherschutz gewünscht sind, ist hier die Verallgemeinerungswirkung sowohl antragsabhängig als auch personell abhängig, also doppelt individualisiert. Wo ein am Sachproblem orientiertes Verfahren not tat, führt Privatisierung der Rechtsverfolgung voraussehbar zur Minimierung der gewünschten Verallgemeinerungswirkung. Ob die Antragsberechtigung der Verbraucherverbände in den Verfahren nach $\oint_{13}$ dem entgegenwirken kann, ist jedenfalls mitentscheidend ein Problem der finanziellen Ausstattung der Prozeßührungsfonds der staatlich geförderten Verbände und Verbraucherzentralen und der Entwicklung von Strategien zur Durchführung von Musterprozessen.

Die privatrechtliche Orientierung setzt sich auch in der Form partieller Bereichsausnahmen für öffentliche Versorgungsunternehmen durch $(\$ 23)$ : Nicht eine einheitliche Regelung, die die Wirksamkeit von Verbraucherschutzpolitik vom öffentlichrechtlich oder privat-rechtlich organisierten Zuteilungsmechanismus ${ }^{11}$ unabhängig macht, sondern Ermächtigungsnormen, nach denen der Wirtschaftsminister gemäß der Generalklausel des $\$ 9$ verfahren kann.

Die endgültige Beurteilung des Gesetzes zur Regelung des Rechts der allgemeinen Geschäftsbedingungen hängt von einer Erfolgskontrolle ab, welche die Auswirkungen der Veränderung der Struktur des materiellen Rechtsprogramms, die Reaktion auf die gesetzliche Normierung durch Verwender und Verbände und auf der Konsumentenseite die Auswirkungen auf die Wahrnehmung von Rechtspositionen zu untersuchen hat. Die Probleme von Zugangssperren der Rechtswahrnehmung werden durch das gewählte Verfahren auch nicht ansatzweise thematisiert.

Aufgrund der Disparität zwischen materiellrechtlicher und verfahrensrechtlicher Programmstruktur läßt sich allerdings schon jetzt vermuten, daß selbst der gegenüber möglichen Problemlösungsansätzen schon reduzierte Anspruch einer Verallgemeinerungswirkung nicht realisiert werden wird, sondern daß dessen Einlösung allein von der Reaktion der AGB verwendenden Unternehmen und der Wahrnehmung von Handlungschancen und der Entwicklung von Handlungsstrategien durch die Konsumentenverbände abhängig ist.

Dieter Hart

Io Bericht des Rechtsausschusses vom 23. 6. 1976 Drucksache 7/5422, S. I3-

it So aber Bundestagsdrucksache $7 / 5422$, S. 4. 